\title{
Estimation of Heritability of Laryngeal Hemiplegia in the Thoroughbred Horse by Gibbs Sampling
}

\author{
Takayuki IBI $^{1 *, 2}$, Takeshi MIYAKE ${ }^{1}$, Seiji HOBO ${ }^{3}$, Hironori OKI $^{3}$, Nobushige ISHIDA ${ }^{3}$ and \\ Yoshiyuki SASAKI ${ }^{1}$ \\ ${ }^{1}$ Division of Applied Bioscience, Graduate School of Agriculture, Kyoto University, Sakyo-ku, Kyoto, 606-8502, \\ ${ }^{2}$ Agura Farm, Sakitama 3-833, Kuroiso, Tochigi, 325-0033 and ${ }^{3}$ Equine Research Institute, Japan Racing \\ Association, 321-4, Tokami-cho, Utsunomiya City, Tochigi 320-0856, Japan
}

\begin{abstract}
Laryngeal Hemiplegia (LH) leads to a reduction in performance and because of it many promising racehorses have been forced to end their racing career. Therefore it is important for breeding good racehorses to estimate the heritability of LH. In this study, the computer program was developed based on the Bayesian analysis with Gibbs sampling for estimating the heritability of categorical traits assuming liability. A total of 706 records with LH-grade in Thoroughbreds aged 2 to 5 years were assigned for the genetic analysis. LH-grades consisted of five severity classes from 0 to 4. Racehorse breeders are often interested in whether the genetic effect controlling a complex disorder is present in the population. To answer this question, the binary trait analysis would be also useful. The heritability of LHgrade in the Thoroughbred horse was then also estimated as a binary or as a categorical trait. The mode values of the posterior distributions of heritability were 0.23 and 0.20 for the binary and the categorical trait, respectively. The fact that in the Thoroughbred population studied LH is at least partially controlled by genetic factors leads to the suggestion that when applying adequate breeding measures the prevalence of $L H$ will be able to be reduced.

Key words: categorical trait, Bayesian analysis, Gibbs sampling, heritability, Laryngeal Hemiplegia
\end{abstract}

\author{
J. Equine Sci. \\ Vol. 14, No. 3 \\ pp. 81-86, 2003
}

Roaring in the Thoroughbred horse is mainly caused by Laryngeal Hemiplegia ( $\mathrm{LH})$ as it causes laryngeal paralysis and a respiratory obstruction of the upper airway. The obvious sign of the defect is a whistling or roaring sounds heard on the inspiratory phase of the respiration. This obstruction leads to a reduction in performance, and many promising racehorses have been forced to end their racing career due to this disorder (JRA 1996). LH is diagnosed by endoscopic examination. According to the criteria described by Hackett et al. (1991), the severity of the disorder was classified under five levels going from unaffected (grade $=0$ ) to severely affected (grade $=4$ ); these severity classes will be called as LH-grade, hereinafter. This disorder is often segregated within (half-sib) families

This article was accepted July 18, 2003

*Corresponding author. suggesting that $\mathrm{LH}$ is controlled genetically. In addition, for a long time LH has been suspected of having a genetic basis as mentioned by Cahill and Goulden (1987) and Poncet et al. (1995), but definite proof of its hereditary nature is lacking. Therefore it is important for breeding good racehorses to clarify the extent of the genetic contribution, e.g., by estimating the heritability of LH. Dependent upon the results of genetic analysis, appropriate selection programs for $\mathrm{LH}$ will be developed.

The concept of liability proposed by Wright (1934) is one of the most widely used approaches for genetic analyses of categorical traits such as LH, as shown in Pirchner (1983) and Falconer and Mackay (1996). This concept is based on an underlying variable (liability) which has a continuous distribution. A response in a given category is observed, if the actual value of liability falls between the thresholds defining the appropriate category (Fig. 1). In the case of binary 

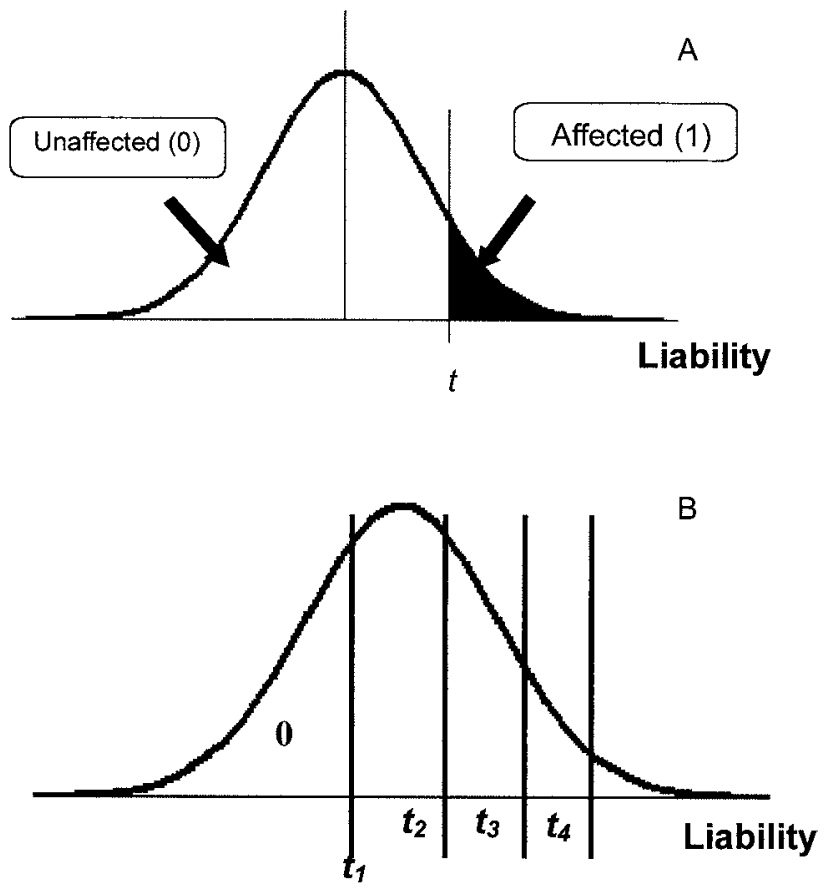

Fig. 1. The concept of liability (A: binary trait, B: categorical trait).

traits where the health status is classified under only two categories, e.g, not affected (classified as 0 ) and affected (classified as 1) and only one threshold exists (Fig. 1A). When the LH-grade is classified under five categories (from 0 to 4 ), number of the thresholds is four (Fig. $1 \mathrm{~B})$. For example, if the liability of an individual is below $t_{1}$, the phenotype is classified as 0 . If it is between $t_{1}$ and $t_{2}$, the phenotype is classified as 1 .

Based on the concept of liability, many statistical methods have been proposed to estimate variance components for categorical traits. Anderson and Aitkin (1985) suggested a maximum likelihood approach for a binary threshold model, which had difficulties in handling the correlation between random effects (e.g. pedigree structure of different animals) that is common in genetic analyses. An approximate method based on Bayesian the approach using a maximum a posteriori (MAP) estimation was proposed for the genetic analysis in animal breeding by Gianola and Foulley (1983), Harville and Mee (1984) and Foulley $e t$ al. (1987). However, the bias of the estimates could be significant (Mayer 1995). Sorensen et al. (1995) and Hoeschele and Tier (1995) proposed a Bayesian approach using Gibbs sampling (Geman and Geman, 1984; Gelfand and Smith, 1990), where the applied approximations would be smaller than those in the MAP estimates and able to handle complex pedigrees, but the convergence to obtain solutions was generally very slow, especially for the threshold parameters. Considering the characteristics of the methods mentioned, the Bayesian approach with Gibbs sampling seems the most appropriate in estimating genetic parameters for categorical traits in animal breeding.

In this study, the computer program, GSTM (computer program for genetic analysis with Gibbs Sampling for Threshold Models), was developed based on the Bayesian analysis with Gibbs sampling. The reliability of the GSTM program was tested using simulated data. The heritability of the LH-grade in the Thoroughbred horse was then estimated with GSTM as well as a binary or as categorical trait.

\section{Materials and Methods}

\section{Development of the GSTM program}

The GSTM program is based on the approach proposed by Sorensen et al. (1995) that assumed a sire model as the genetic model. The GSTM program expanded the genetic model to an animal model. Conditional posterior density functions, which were needed to construct the polygenic model, were obtained with the program package MAGGIC (Janss, 1998). According to the discussion of Hoeschele and Tier (1995) and Moreno et al. (1997), the so-called herd-year-season (HYS) effect was treated as a random effect. To treat it as a random effect is effective because its means are zero and therefore this does not change the normal shape of the liability distribution. Effect with only two levels (e.g. sex) was treated as covariates. In categorical trait analyses, it is generally rather difficult to obtain convergence compared with continuous trait analyses because the number of unknown parameters increases (e.g. number of liability values for each individual and threshold values). Sorensen et al. (1995) also discussed the difficulty of convergence for thresholds. That was the reason why a large number of samplings were applied for the categorical trait analysis.

In the analysis using Gibbs sampling, many samples for unknown parameters were generally obtained beginning with initial values. By repeating Gibbs sampling cycles, the samples obtained for a parameter 


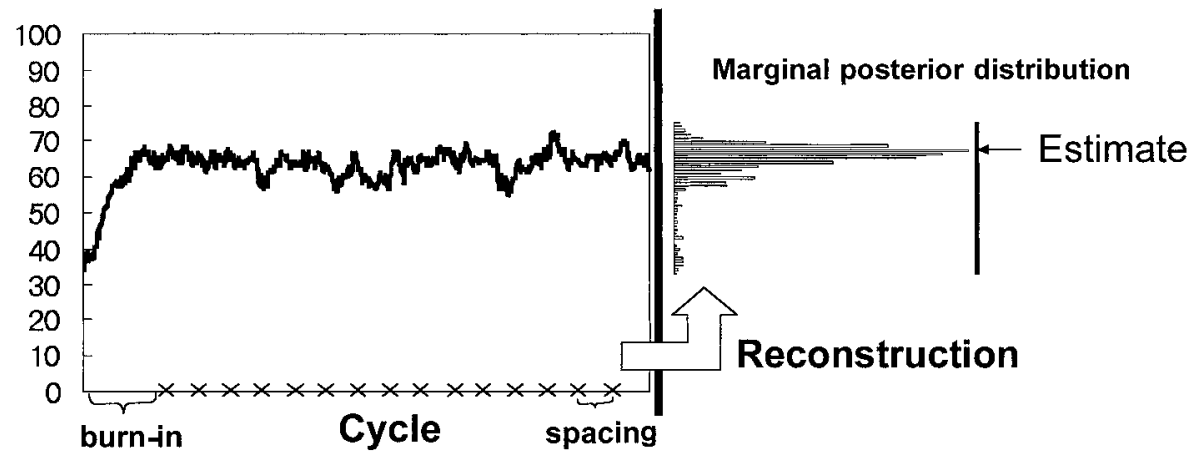

Fig. 2. Outline of the analysis by means of Gibbs sampling.

Table 1. Estimates of heritability in simulation examples with various true heritabilities

\begin{tabular}{cc}
\hline True values & Estimates* \\
\hline 0.0 & $0.02 \pm 0.03$ \\
0.1 & $0.11 \pm 0.06$ \\
0.2 & $0.18 \pm 0.06$ \\
0.3 & $0.31 \pm 0.07$ \\
0.4 & $0.41 \pm 0.04$ \\
0.5 & $0.50 \pm 0.06$ \\
\hline
\end{tabular}

* Mean \pm standard deviation of the mode values for 20 replicates.

generally tended towards a certain stationary phase (i.e. the graph tends to become horizontal with some fluctuations), which means convergence was achieved. The period before achieving the stationary phase is called the burn-in period. The marginal posterior distribution (density) for the parameter was constructed with the data for the stationary phase. The mode (or mean) is chosen as estimation for the parameter. To enhance the randomization of each sample, spacing was applied in order to build the posterior density. An outline of the analysis using Gibbs sampling is shown in Fig. 2.

\section{Simulation example}

Before the heritability of LH-grade was estimated, the GSTM program was tested with a simulated half-sib population. 50 sires were randomly mated with 20 dams each with one offspring per dam. The total number of animals in this population was therefore 2,050 .
In this population, the following polygenic model for threshold traits with three severity classes (from 1 to 3 ) was used;

$$
\begin{aligned}
& L_{i}=u_{i}+e_{i} \text {, and } \\
& Y_{i}=1\left(L_{i} \leq t_{1}\right), \\
& Y_{i}=3\left(\mathrm{t}_{2}<L_{i}\right)
\end{aligned} \quad Y i=2\left(t_{1}<L_{i} \leq t_{2}\right) \text { and }
$$

where $L_{i}$ is the liability of the individual $i, u_{\mathrm{j}}$ is the breeding value $\left(N\left(0, \mathrm{~A}^{2}{ }_{u}\right)\right), e_{i}$ is the residual effect $\left(N\left(0, \mathrm{I}^{2}{ }_{e}\right)\right), Y_{i}$ is phenotype, $t_{x}$ is threshold levels $(x=1$ and 2), $\mathrm{A}$ is numerator relationship matrix, $\sigma^{2}{ }_{u}$ is genetic variance and $\sigma^{2}{ }_{e}$ is residual variance. Heritability $\left(h^{2}\right)$ is defined as $\sigma^{2}{ }_{u} / \sigma^{2}{ }_{u}+\sigma^{2}{ }_{e}$ ). Threshold values were fixed at $t_{1}=0$ and $t_{2}=1$ S.D. unit of liability, respectively. The heritabilities were assigned from 0.0 to 0.5 with 0.1 spacing. For each heritability setting, 20 replicates were generated.

These simulated populations were analyzed with the GSTM program by using model (1) in order to estimate the heritabilities. In the analysis with Gibbs sampling, the total number of samplings was 210,000 and the first 10,000 samples were discarded as the burn-in. Thereafter, samples were stored every 100 iterations (spacing) so that the number of samples used for the construction of the posterior density was 2,000. For each replicate, the mode value of the constructed posterior density was chosen as the estimate. Results are shown as the mean and the standard deviation of the mode values obtained from the twenty replicates.

Table 1 shows the estimated heritabilities for the simulated populations. In all cases, the heritabilities were estimated unbiased, suggesting that the application of the GSTM program for the practical data would be effective. 


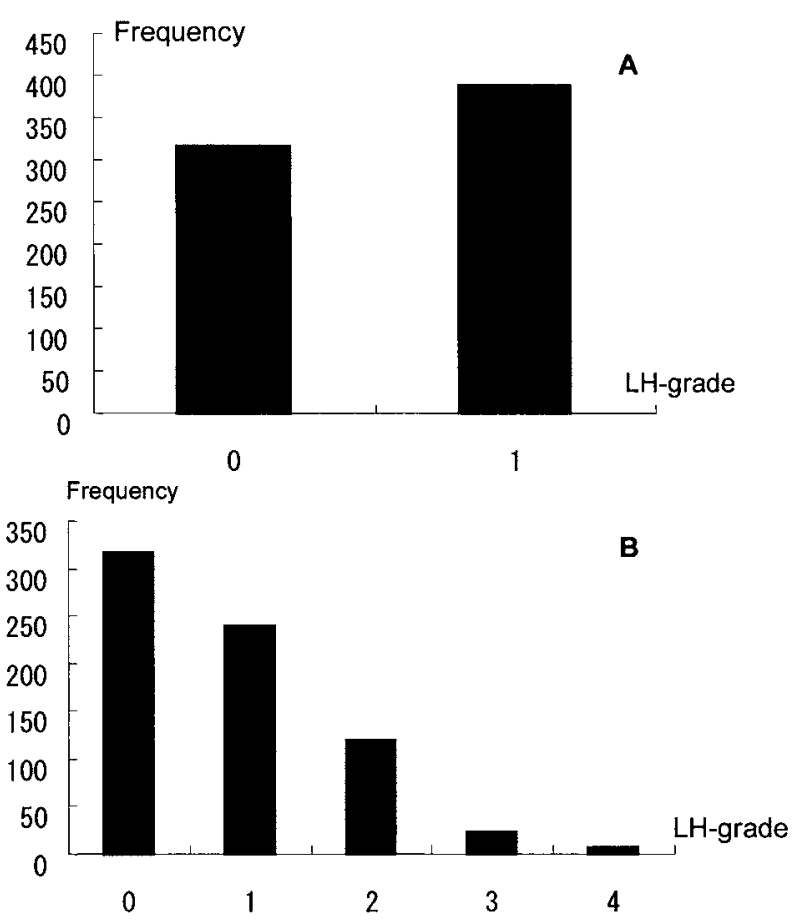

Fig. 3. Frequency distribution of the LH-grade in the Thoroughbred horse (A: binary trait, B: categorical trait).

\section{LH-grade in racehorse}

For the genetic analysis 706 records of LH-grade of 2 to 5 year old Thoroughbreds that were recorded at The Racehorse Clinic at JRA's Ritto Training Center, were available. Only stallions that had more than two offspring with records were used in the analysis. If an animal had several diagnoses in its lifetime, the largest value was kept for the analysis. Pedigree information was traced back to the great-grandparents.

As mentioned above, the LH-grades consisted of five severity classes going from 0 to 4 (Hackett et al. 1991; Hobo et al. 1995). Racehorse breeders are often interested in whether a genetic effect controlling a complex disorder is present in the population. To answer this question, the binary trait analysis would be also useful. Two genetic analyses were therefore performed: i) where the LH-grade was treated as a categorical trait with five classes, ii) where the LH-grade was treated as a binary trait by differentiating only between unaffected ( $\mathrm{LH}$-grade $=0)$ and affected ( $\mathrm{LH}-$ grade $>0)$. Fig. 3 shows the frequency distributions of the LH-grade for the binary and the categorical traits.

A preliminary analysis with the SAS CATMOD procedure (SAS Institute Inc. 1985) revealed that the sex effect was significant $(p<0.05)$ in LH -grades. Therefore, the sex effect was included in the model. The variance components needed to estimate the heritability were obtained by analyzing the following model:

$$
\begin{array}{ll}
L_{i j}=S E X_{i}+u_{j}+e_{i j}, \text { and } & \\
Y_{i j}=0\left(L_{i j} \leq t_{1}\right), & Y_{i j}=1 \quad\left(t_{1}<L_{i j} \leq t_{2}\right), \\
Y_{i j}=2\left(t_{2}<L_{i j} \leq t_{3}\right), & Y_{i j}=3\left(t_{3}<L_{i j} \leq t_{4}\right) \text { and } \\
Y_{i j}=4\left(t_{4}<L_{i j}\right) &
\end{array}
$$

when the disorder was classified as a categorical trait, and

$$
\begin{aligned}
& L_{i j}=S E X_{i}+u_{j}+e_{i j}, \text { and } \\
& Y_{i j}=0\left(L_{i j} \leq t\right) \text { and } Y_{i j}=1\left(t<L_{i j}\right)
\end{aligned}
$$

when the disorder was classified as a binary trait, where $S E X_{i}$ is the effect of the $i$ th sex. This effect was treated as covariate.

In the Gibbs sampling analysis, the total number of Gibbs samplings was 10,000,000. The optimal burn-in and the spacing values were determined based on the method of Raftery and Lewis (1996). In addition to the mode values of the constructed posterior density the mean and median were calculated as well.

\section{Results and Discussion}

In Table 2, the mean, mode and median of the posterior distribution of the heritabilities as well as the burn-in, the spacing and the number of samples used for the construction of the posterior distribution are shown for the binary and the categorical trait analysis. The marginal posterior distributions obtained for the heritability for the LH-grade are shown in Fig. 4. The

Table 2. Estimates of heritability for LH-grade

\begin{tabular}{lcccccc}
\hline & Burn-in & Spacing & Samples* & Mean & Mode & Median \\
\hline Binary trait & 4,496 & 17,960 & 557 & 0.3627 & 0.2326 & 0.3290 \\
Categorical trait & 2,979 & 23,863 & 419 & 0.3042 & 0.2040 & 0.2721 \\
\hline
\end{tabular}

* Number of samples used for the construction of the posterior distribution. 

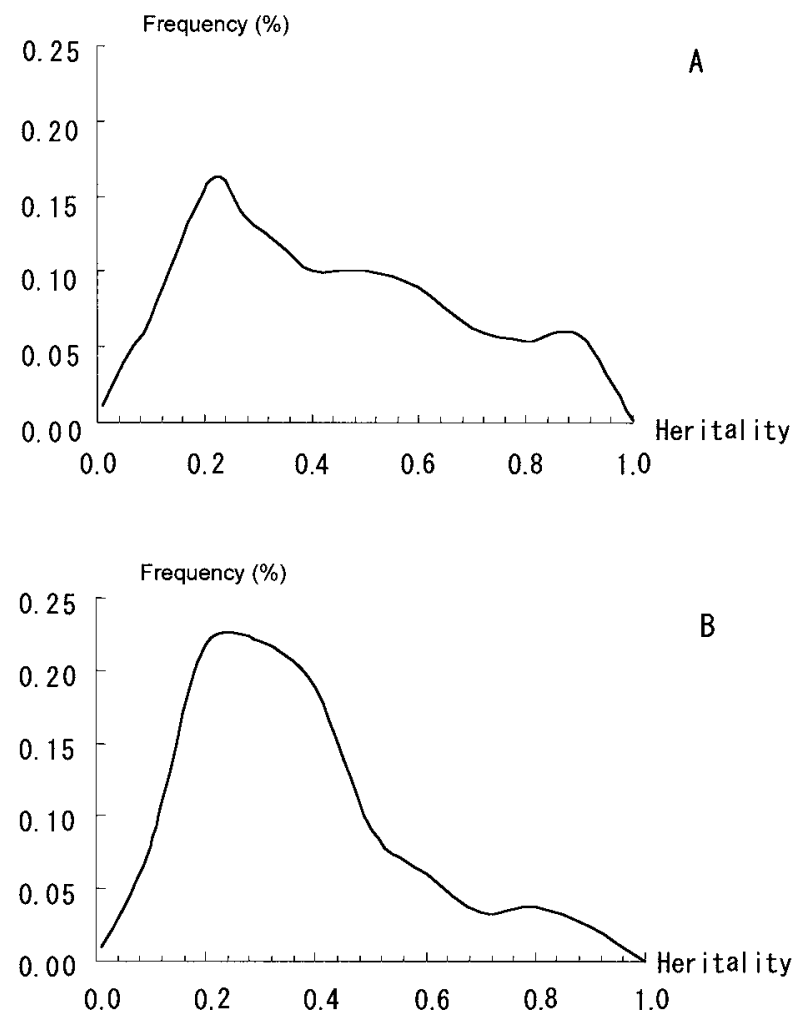

Fig. 4. The constructed marginal posterior distributions for the heritability of the LH-grade in the Thoroughbred horse (A: binary trait, B: categorical trait)

mode values of the posterior distributions were 0.23 and 0.20 for the binary and the categorical trait, respectively (Table 2 ). The densities of both posterior distributions of the heritability equal zero were very low, suggesting that the estimated heritability of LHgrade was at least not zero. The significance of the two mode values can be tested by the method of Janss $e t a l$. (1995), i.e. the mode value is significant if the density at the mode value is 20 -fold (i.e. $p=0.05$ ) larger than density at the parameter value of zero. Following this suggestion, our mode values were revealed to be significant at a level of $p<0.10$ in the binary trait analysis, and $p<0.05$ in the categorical trait analysis. Therefore, it is suggested that Laryngeal Hemiplegia is genetically controlled to some extent in the Thoroughbred population.

In this study, the estimated heritability for the LHgrade in the binary trait analysis was slightly higher than the one in the categorical trait analysis. In addition, in the categorical trait analysis, the density distribution between the heritability of 0.2 and 0.4 was flat. This observation suggests that the heritability of the LHgrade may be higher.

The fact that in the studied Thoroughbred population LH is at least partially controlled by genetic factors leads us to suggest that when applying adequate breeding measures the prevalence of $\mathrm{LH}$ will be able to be reduced. An important part of that selection program would be the systematic screening of breeding stallions and mares as well as their offspring by endoscopic examination. Based on these data breeding values could be calculated and used as a selection criterion. Furthermore, if from each horse examined hair a root or blood sample is collected in order to obtain DNA, molecular genetic approaches could be started to find the gene(s) or QTLs that control LH.

\section{Acknowledgments}

We are grateful to Dr. C. Gaillard for helpful comments, advice on the manuscript and for polishing the English.

\section{References}

1. Andersonand, D.A. and Aitkin, M. 1985. Variance component models with binary response: interviewer variability. J. R. Stat. Soc. B. 47: 203210.

2. Cahill, J.I. and Goulden, B.E. 1987. The pathogenesis of equine laryngeal hemiplegia-a review. N. Z. Vet. J. 35: 82-90.

3. Falconer, D.S. and Mackay, T.F.C. 1996. Introduction to quantitative genetics, 4th ed., Longman, Harlow.

4. Foulley, J.L., Gianola, S.D., and Hoeschele, I. 1987. Empirical Bayes estimation of parameters for $n$ polygenic binary traits. Genet. Sel. Evol. 19: 197-224.

5. Gelfand, A.E. and Smith, A.F.M. 1990. Samplingbased approaches to calculating marginal densities. J. Am. Stat. Assoc. 85: 398-409.

6. Geman, S. and Geman, D. 1984. Stochastic relaxation, Gibbs distributions and Bayesian restoration of images. IEEE Trans. Pattn. Anal. Mach. Intell. 6: 721-741. 
7. Gianola, D. and Foulley, J.L. 1983. Sire evaluation for ordered categorical data with a threshold model. Genet. Sel. Evol, 15: 201-224.

8. Hackett, R.P., Ducharme, N.G., and Fubini, S.L. 1991. The reliability of endoscopic examination in assessment of arytenoids cartilage movement in horses Part 1; subjective and objective laryngeal evaluation. Vet. Surg., 20: 174-179.

9. Harville, D.A. and Mee, R.W. 1984. A mixed-model procedure for analyzing ordered categorical data. Biometrics 40: 393-408.

10. Hobo, S., Matsuda, Y. and Yoshida, K. 1995. Prevalence of upper respiratory tract disorders detected with a flexible videoendoscope in thoroughbred racehorses. J. Vet. Med. Sci. 57: 409413.

11. Hoeschelle, I. and Tier, B. 1995. Estimation of variance components of threshold characters by marginal posterior modes and means via Gibbs sampling. Genet. Sel. Evol. 27: 519-540.

12. Janss, L.L.G. 1998. pp.459-460. MAGGIC: A package of subroutines for genetic analysis with Gibbs sampling, In: Proc. 6th World Congr. Genet. Appl. Livest. Prod. UNE. Armidale NSW, Australia. Vol 27.

13. Janss, L.L.G., Thompson, R., and Van Arendonk J.A. M. 1995. Application of Gibbs sampling for inference in a mixed major gene-polygenic inheritance model in animal populations Theor. Appl. Genet. 91: 1137-1147.

14. JRA. 1996. Upper respiratory tract disorders of racing horses, The Racehorse Clinic at JRA's Ritto
Training Center.

15. Mayer, M. 1995. Inequality of maximum a posteriori estimators with equivalent sire and animal models for threshold traits. Genet. Sel. Evol. 27: 423-435.

16. Moreno, C., Sorensen, D., Garci'a-Corte's, L.A., Varona, L. and Altarriba, J. 1997. On biased inferences about variance components in the binary threshold model. Genet. Sel. Evol. 29: 145160.

17. Pirchner, F. 1983. Threshold traits. In: "Population Genetics in Animal Breeding", Ed.2, pp.285-294, Plenum Press, NY and London.

18. Poncet, P.A., Montavon, S., Gaillard, C., Barrelet, F. Straub, R., and Gerber, H. 1989. A preliminary report on the possible genetic basis of laryngeal hemiplegia. Equine Vet. J. 21: 137-138.

19. Raftery, A.E. and Lewis, S. 1996. Implementing MCMC, in Markov Chain Monte Carlo in Practice, (eds W. R. Gilks, S. Richardson and D. J. Spiegelhalter). Chapman \& Hall, London, pp. 115-130.

20. SAS Institute Inc., 1994. pp.405-518. SAS/STAT User's Guide, Version 6.4. Volume 1. Cary, NC.

21. Sorensen, D.A., Anderson, S., Gianola D., and Korsgaard, I. 1995. Bayesian inference in threshold models using Gibbs sampling. Genet. Sel. Evol. 27: 229-249.

22. Wright, S. 1934. An analysis of variability in number of digits in an inbred strain of guinea pigs. Genetics 19: 506-536. 